\title{
L'intégration Et La Pratique Des Démarches De La Didactique De La Grammaire Du FLE Dans Les Écoles Secondaires Du District De Kayonza, Au Rwanda: État Des Lieux
}

\author{
Rugigana Aimable ${ }^{1 *}$, Dr. Chokah Milcah ${ }^{2}$, Dr. Caroline C. Oyugi ${ }^{3}$ \\ ${ }^{1}$ Assistant Lecturer (French Didactics), University of Rwanda-College of Education, Department of Humanities and Language-Education, P.O. Box: 55 \\ Rwamagana-Rwanda \\ ${ }^{2}$ Senior Lecturer, Kenyatta University, Department of Literature, Linguistics and Foreign Languages, P.O. Box 43844 Nairobi-Kenya \\ ${ }^{3}$ Lecturer, University of Nairobi, Department of Literature -Sub-Department of French, P.O. Box 30197, GPO, Nairobi, Kenya
}

DOI: $10.36348 /$ sijll.2020.v03i08.003

| Received: 19.08 .2020 | Accepted: 26.08 .2020 | Published: 29.08 .2020

*Corresponding author: Rugigana Aimable

\section{Abstract}

L'observation de la pratique de classe et l'analyse didactique des programmes et des manuels de français d'avant l'introduction du curriculum basé sur les compétences au Rwanda, montre que la didactique de la grammaire de français suivait une démarche déductive qui met au centre de tout enseignement l'enseignant, le rôle de l'apprenant relégué au second plan. L'an 2008 fait que le français passe de la langue d'instruction à la langue objet d'enseignement, supplantée par l'anglais. L'intérêt d'apprendre et d'enseigner cette langue va sensiblement régresser. Aujourd'hui, pour relever l'enseignement efficace du français et de la grammaire en particulier, les efforts doivent converger vers l'intégration et la pratique des nouvelles démarches de la didactique de la grammaire. Leur coexistence n'y est pas pour rien car la résistance au changement persiste suite au degré de connaissance et d'utilisation de ces démarches et à l'attitude des enseignants et des apprenants face à la situation nouvelle. Notre recherche à la fois qualitative et quantitative, avait pour objectif de présenter la situation actuelle de la didactique de la grammaire du FLE dans les écoles secondaires au Rwanda, en vue de proposer une meilleure pratique de ses démarches. Elle a focalisé sur les élèves de la $2^{\text {ème }}$ et de la $5^{\text {ème }}$ année, et leurs enseignants des six écoles sélectionnées du district de Kayonza au Rwanda. Leurs réponses au questionnaire et à l'entretien ont prouvé que l'enseignement de la grammaire du FLE dans ces écoles reste traditionnelle et déductive .Les démarches inductives et active de découverte sont quasiment inconnues et rarement utilisées, d'où la nécessité d'une formation et une pratique de ces nouvelles démarches pour une didactique efficace de la grammaire en particulier et du français en général.

Keywords: Didactique, démarche, grammaire, FLE, enseignement, situation, coexistence, attitude.

Copyright @ 2020: This is an open-access article distributed under the terms of the Creative Commons Attribution license which permits unrestricted use, distribution, and reproduction in any medium for non-commercial use (NonCommercial, or CC-BY-NC) provided the original author and source are credited.

\section{INTRODUCTION}

L'objet principal de cet article est de présenter la situation actuelle de la didactique de la grammaire du FLE dans les écoles secondaires au Rwanda, en vue de proposer une meilleure pratique de ses démarches. Plus particulièrement cette recherche vise à établir le bilan sur la pratique des démarches de la didactique de la grammaire du FLE dans les écoles secondaires sélectionnées du district de Kayonza. Elle se propose aussi d'examiner le degré de coexistence des trois démarches de la didactique de la grammaire en classe de FLE et de démontrer l'attitude des enseignants et des élèves face aux nouvelles démarches de la didactique de la grammaire au Rwanda
En effet, la didactique de la grammaire oppose les approches inductives et les approches déductives. Les premières, associées à la grammaire nouvelle, se définissent comme une approche où, au contact préalable d'exemples, les élèves induisent une règle, alors que les approches déductives, plus traditionnelles, sont inversement associées à une stratégie d'enseignement utilisée pour amener les élèves à s'exercer après qu'il y ait eu explication d'une règle.

La démarche active de découverte (DADD), quant à elle, les élèves prennent, d'abord, conscience de la difficulté grammaticale par l'observation du phénomène à partir d'un corpus. Ensuite, ils manipulent les éléments constitutifs du corpus qui représentent le phénomène en classant, en modifiant des énoncés. Les hypothèses formulées permettent de rédiger les lois ou 
les règles qui seront vérifiées par les élèves à partir de différents ouvrages de références à leur disposition. Enfin, cette phase est suivie de la phase d'application de ces règles dans différents contextes linguistiques. Ils réinvestiront, par la suite, ces connaissances sous l'égide de l'enseignant dans les activités de réception et de production [11].

Une simple lecture de l'histoire de l'évolution des méthodologies de la didactique des langues en général et du français en particulier, montre que chaque méthodologie suivait une démarche précise dans l'enseignement de la grammaire. A part la grammaire traduction aussi connue comme la méthodologie traditionnelle qui a connu une démarche déductive, les autres méthodologies, de la méthodologie directe à la méthodologie orale, ont suivi une démarche inductive.

La Méthodologie Audio-visuelle (MAV) qui a dominé en France entre les années 1960 et 1970 a introduit dans l'enseignement du français une démarche active de découverte. Le déroulement de la leçon suivait les étapes telles que présentation ou explication, répétition ou mémorisation, exploitation ou fixation, transposition ou appropriation. Cette démarche sera raffinée avec l'Approche communicative des années 70 en réaction contre la méthodologie audio - orale et la méthodologie audio-visuelle. Pour Porcher [6], dans l'Approche Communicative, l'enseignement de la grammaire était implicite: au début, elle est inductive, puis elle est conceptualisée, expliquée et, finalement, elle suit la règle (déduction).

La démarche active de découverte en grammaire cherche à faire comprendre les grandes régularités du fonctionnement de la langue. Elle fait appel aux capacités d'observation, d'expérimentation, de raisonnement et d'argumentation des élèves. Souvent associée à une démarche inductive, elle ne s'y réduit pas, car elle fait aussi appel au raisonnement déductif [11].

L'Approche Communicative débouchera dans les années 1990 à l'approche actionnelle [5]. Pour elle, l'enseignement de la langue est basé sur les tâches à réaliser au sein d'une activité globale. Dans bien des pays du monde, la didactique de la grammaire du français a connu des réformes dans les programmes d'enseignement du français, langue d'enseignement ou langue étrangère. C'est l'exemple des pays tels que la France, le Canada, la Belgique et la Suisse.

A partir des années 1980, l'accent est mis sur la communication. L'élève apprend la grammaire en situation de communication; de lecture ou d'écriture. La grammaire n'est pas enseignée isolée mais dans le contexte. Il joue un rôle participatif et peut s'exprimer librement. En 1995, au Québec, la grammaire traditionnelle est supplantée par la grammaire nouvelle. Elle devient plus explicite et systématique et voit ses contenus et ses démarches révisés et redéfinis. Elle préconise la démarche active de découverte qui permet à l'élève d'observer un phénomène grammatical afin d'en déduire la règle lui-même. Après une dizaine d'année les chercheurs comme Nadeau et Fisher se demandaient si la grammaire traditionnelle et la grammaire nouvelle coexistaient encore en classe de français et si les enseignants avaient réussi à vivre cette transition [8].

En 2000, un nouveau programme de français permet à l'enseignant de placer l'élève dans une situation de communication authentique. Celui-ci fait recours aux connaissances sur la langue, les textes et la culture. Il acquiert non seulement des connaissances linguistiques mais aussi la façon de les utiliser en dehors de la classe.

Dans le contexte rwandais, durant les trois dernières décennies, l'enseignement du français au Rwanda suivait des programmes élaborés par le ministère de l'éducation, s'inspirant bien sûr des programmes d'autres pays francophones et aujourd'hui du Cadre Européen Commun de Références pour les Langues (CECRL). Chaque programme faisait l'objet de la rédaction des manuels de français pour les élèves et pour les enseignants. Dans cet article, nous nous sommes bornés sur les manuels des écoles secondaires, lesquelles écoles ont fait l'objet de notre recherche.

En effet, les générations des années 90 jusqu'aux années 2007 exploitaient les manuels tels que Comprendre et s'exprimer (fascicule 1, 2,3) pour la première année secondaire, Comprendre et s'exprimer (fascicule 4,5,6) destiné à la deuxième année de l'école secondaire, La condition féminine et L'Epopée Mandingue pour la troisième année de l'école secondaire. Ceux qui ont fréquenté le cycle supérieur de l'école secondaire entre 1990 et 2008, toutes options confondues, ont sans doute une bonne mémoire des Dossiers étudiés tels que Le Dossier 6: L'eau et la sécheresse, le dossier 7: Alcool: mythes et réalité, Dossier 8: Amour, dot et mariage, etc. Une analyse des contenus et des démarches d'enseignement consignées dans ces programmes et manuels montre que l'objectif visé était de conduire les élèves à pouvoir comprendre et s'exprimer. La grammaire dans ce contexte était intégrée dans l'analyse des textes et «progressivement les textes deviennent plus complexes, les exercices font appel à plus de connaissances linguistiques de telle sorte que les élèves guidés par leurs professeurs, pourront réellement, de ce cours, comprendre des conversations, des textes français et s'exprimer dans cette langue» (comprendre et s'exprimer, tome4:3). Dans ces manuels, l'enseignement de la grammaire suivait une démarche déductive et explicite. Pour Coste \& Galisson: La grammaire explicite est fondée sur l'exposé et l'explicitation des règles par le professeur, suivi d'applications conscientes par les élèves [9]. 
Depuis le premier fascicule des manuels de la première année, l'insistance est mise sur la grammaire. A partir d'un texte étudié, il est expliqué une règle de grammaire, suivi d'un exercice d'application par les élèves. Cette grammaire suit une démarche déductive car les élèves trouvent, déjà, dans leurs manuels une description grammaticale et des encadrés qu'ils doivent noter dans leurs cahiers, mémoriser et appliquer sans aucun effort de découverte. L'enseignement magistral de la grammaire devrait se terminer dans les trois premières années de l'école secondaire, on ne reviendrait sur les structures grammaticales, pour les approfondir que dans les dossiers pour la quatrième année et la cinquième année.

Outre les règles de grammaire exploitées dans trois premières années du secondaire, le programme visait surtout l'expression et la compréhension orale et écrite. Mais à partir de la quatrième année, l'enseignant commençait à pendre le rôle de facilitateur. L'explication de ce phénomène est très simple, les élèves avaient commencé à apprendre le français depuis la quatrième année de l'école primaire et il n'y avait pas d'autres langues étrangères en concurrence. Le terrain était préparé; il ne s'agissait que d'améliorer. Le dossier, dans cet angle proposait un éventail aussi large que possible de textes et d'activités susceptibles de contribuer à l'amélioration communicative de l'élève.

Depuis Octobre 2008, le français langue d'enseignement devient langue - objet d'enseignement, supplantée par l'Anglais. L'enseignement primaire, secondaire, et universitaire est exclusivement dispensé en anglais. Mais le français garde son statut de coofficialité. Selon la Constitution de la République du Rwanda de 2003 telle que révisée en 2015, dans son article no 8 : « La langue nationale est le kinyarwanda. Les langues officielles sont le kinyarwanda, l'anglais, le français et le swahili. ». Depuis ce changement non seulement le statut linguistique du français a été affecté mais aussi des représentations que les Rwandais ont de cette langue européenne ont influencé l'attitude des enseignants et des apprenants face à cette langue.

Par ailleurs, un retour en arrière montre que depuis 1996, plusieurs réformes sur le curriculum ont été faites et le français n'en est pas sorti indemne. Consécutivement la réforme de 1996, concernait le cycle inferieur de l'école secondaire et celle de 1997 concernait l'école primaire dans toutes les matières. La révision du programme de français de 1999, concernait le cycle supérieur scientifique.

Cependant, une révision plus nette et plus systématique devrait avoir lieu en 2003, incluant le cycle supérieur. C'était un plan éducatif de 6 ans (qui va de 2003 à 2008). Durant toute cette période, le français était langue d'enseignement et gardait entre 3heures et 6 heures par semaine. La révision du programme en Novembre 2008, a fait que le français qui était jusqu'alors obligatoire et examiné de l'école primaire et à l'école secondaire (5heures tout le cycle complet de l'école primaire) se trouve décliné tant pour l'école primaire (3heures au cycle inferieur et 2 heures au cycle supérieur)que pour l'école secondaire (2 heures par semaine au tronc- commun et au cycle supérieur, 9heures pour la combinaison FrançaisAnglais-Kinyarwanda et 4 heures pour la combinaison Pédagogie -langues). Dans ce contexte, l'enseignement $\mathrm{du}$ français est obligatoire et ne fait pas parti de l'examen d'état.

De 2010 à 2015, le français ne figure pas sur la liste des langues enseignées à l'école primaire ; il garde le rôle facultatif à l'école secondaire (2heures par semaine durant tout le cycle complet) et enseigné dans quelques combinaisons : Français-Anglais -Education, Kinyarwanda-Français -Education, Traduction et interprétariat, dans certaines universités et Instituts supérieurs du Rwanda.

Depuis 2012, le programme de français stipule, dans l'approche méthodologique d'enseignement de la grammaire du français au Rwanda, que la didactique de la grammaire se fait en situation de communication. Elle doit être une grammaire qui permet à l'élève de découvrir les règles, de les comparer et de les réutiliser dans des phrases. Les supports pédagogiques peuvent être des dialogues, des récits, des saynètes, des dessins, des schémas ou des images.

On a dans cette approche, une amorce de la démarche inductive et pourquoi pas l'annonce de la démarche active de découverte dont les exigences seront précisées dans le nouveau programme de français de 2013. Pour lui, la participation active et l'interaction des élèves permettent de développer la compétence à communiquer en situation authentique. La didactique de la grammaire suit celle du FLE. Elle est fondée sur des compétences génériques et des compétences langagières où l'enseignant est un facilitateur qui doit analyser les besoins des apprenants, la nature de l'apprentissage en rapport avec la situation.

Bien que cette approche ne nomme pas la démarche dont il est question, on le devine et l'inauguration du nouveau programme basé sur les compétences, en Janvier 2016 a révélé, la nécessité de l'utilisation des nouvelles démarches dans l'enseignement du français au Rwanda.

En sus de cela, le français est désormais un cours obligatoire depuis le cycle supérieur de l'école primaire jusqu'à la fin des études secondaires dans les options littéraires et d'autres options qui l'ont choisi. Ce programme embrasse les nouvelles méthodes d'enseignement dont l'approche basée sur les compétences et centrée sur l'apprenant. Pour ce faire, l'idée de compétence n'affirme rien d'autre que le souci 
de faire des savoirs scolaires des outils pour penser et agir, au travail et hors travail [10].

L'exécution de ce programme et plus spécifiquement l'enseignement de la grammaire nécessite l'adoption et la pratique efficiente des nouvelles démarches de la didactique de la grammaire $\mathrm{du}$ français. Si ces démarches viennent de faire une vingtaine d'années, elles ne sont pas encore en vogue dans l'enseignement du français au Rwanda. Ainsi avons-nous été curieux d'explorer leur intégration dans la didactique de la grammaire française au Rwanda, une composante de la langue que nous considérons comme base pour maîtriser les autres compétences linguistiques et communicatives en Français.

\section{METHODOLOGIE}

Notre recherche à la fois qualitative et quantitative, en ce sens qu'un certain nombre de questionnaires a été soumis aux enseignants et aux élèves pour recueillir leurs précisions sur la pratique des démarches de l'enseignement de la grammaire en classes de FLE au Rwanda. Les données recueillies par questionnaire ont été appuyées par l'entretien individuel avec les enseignants du français et l'observation du déroulement des cours de français. Elle s'est effectuée au Rwanda, dans la province de l'Est, plus précisément dans le district de Kayonza. Ce district connaît des localités purement rurales mais certaines écoles sont situées dans un milieu semi -urbain. Les participants sont, principalement, 10 enseignants du cours de français dans les six écoles secondaires du district de Kayonza qui sont désignées comme $\mathrm{E}_{1}, \mathrm{E}_{2}, \mathrm{E}_{3}, \mathrm{E}_{4}, \mathrm{E}_{5}, \mathrm{E}_{6}$. Ces écoles ont été objectivement choisies suivant leur accessibilité et leur volonté de participer à notre recherche.

Deux écoles sont situées dans un milieu semiurbain et quatre écoles sont dans un milieu purement rural. Une école sur cinq est une école des filles. Ces caractéristiques de notre population nous ont permis de recueillir une variété de données utiles à notre recherche. Puisque nous voulions aussi recueillir les points de vue et la position des bénéficiaires de l'enseignement de langue française (les élèves), nous avons inclus 1341 élèves de la cinquième et de la deuxième année dont le français fait partie de leurs cours. Ainsi la population de notre recherche est -elle constituée de 1351 participants.

Table-1: Nombre des élèves de la deuxième année et de la cinquième année et des enseignants participant à la recherche

\begin{tabular}{|l|l|l|l|l|l|l|l|}
\hline Ecoles & $\mathrm{E}_{1}$ & $\mathrm{E}_{2}$ & $\mathrm{E}_{3}$ & $\mathrm{E}_{4}$ & $\mathrm{E}_{5}$ & $\mathrm{E}_{6}$ & Total \\
\hline $\mathbf{2}^{\text {ème }}$ année & 250 & 130 & 212 & 175 & 165 & 95 & 1025 \\
\hline $\mathbf{5}^{\text {ème }}$ année & 60 & 120 & 63 & 0 & 41 & 32 & 316 \\
\hline Enseignants & 3 & 1 & 2 & 2 & 1 & 1 & 10 \\
\hline Total & 313 & 251 & 277 & 179 & 207 & 128 & 1351 \\
\hline
\end{tabular}

Source: La direction des écoles et les visites physiques dans des classes (1 p. 31)

Dans cette recherche exploratoire, nous avions besoin d'un échantillonnage qui répond aux objectifs de notre recherche. C'est un échantillonnage dans lequel le chercheur choisit les sujets et les sites pour étudier et comprendre le phénomène principal [2]. Les participants à notre recherche ont été objectivement choisis pour constituer l'échantillon de notre recherche [2, 12-14]. Le nombre exact des enseignants et des élèves de la $2^{\text {ème }}$ et de la $5^{\text {ème }}$ a été retenu à partir du Tableau pour Déterminer la taille de l'Echantillon pour une Population donnée [15].

L'échantillonnage délibéré a été utilisé pour sélectionner les enseignants parce qu'ils étaient peu nombreux et étaient experts en matière de la didactique du français en général et de la grammaire en particulier [3]. Ils étaient au nombre de 10.

Les élèves ont été délibérément choisis parce qu'ils étaient des personnes appropriées pour nous donner des informations adéquates sur la situation d'apprentissage de la grammaire en classe de FLE. Nous croyions qu'ils avaient des informations dont nous avions besoin [3].
De la population de 10 enseignants et 1341 élèves des six écoles secondaires du district de Kayonza, 312 participants ont été échantillonnés pour réduire le coût, le temps et l'effort. L'échantillonnage a été fait de telle manière que chaque catégorie des participants était représentée dans l'échantillon.

Pour bien mener notre recherche nous nous sommes servis du questionnaire et de l'entretien individuel et de l'observation de classe. L'entretien individuel, nous a permis de récolter des informations sur les représentations et les attitudes des enseignants sur l'apprentissage de la grammaire en classe de FLE [4]. Aussi nous a- t- il aidés à explorer des aspects encore peu connus, de mettre en évidence des questions et des problèmes importants sur les démarches de la didactique de la grammaire et d'analyser des résultats issus du questionnaire, notamment dans l'idée de saisir le «pourquoi » des réponses.

\section{RESULTATS ET DISCUSSIONS}

L'analyse des réponses obtenues par questionnaire et par entretien avec nos participants nous a conduits aux résultats suivants, classés sous trois 
rubriques: la situation actuelle de la didactique de la grammaire en classe de FLE au Rwanda, la coexistence des trois démarches ainsi que l'attitude des enseignants et des élèves face à cette situation de didactique de la grammaire du FLE.

\section{La situation actuelle de la didactique de la grammaire en classe de FLE au Rwanda}

La situation actuelle de la didactique de la grammaire en classe de FLE au Rwanda est caractérisée par l'expérience des enseignants en général, l'appréciation personnelle du niveau de connaissance de la grammaire française, la signification de la grammaire pour les élèves ,la place de la grammaire pendant le cours, l'importance de la grammaire par rapport à d'autres parties du cours de français, la révision de la démarche à suivre avant la préparation du cours ainsi que l'intégration de la grammaire dans un texte ou un autre document déclencheur ou son isolement.

En effet, la plus haute représentation de l'expérience dans l'enseignement en général est comprise entre 11 et 16 ans avec $60 \%$ des participants, puis viennent ceux qui ont entre 6 et 10 ans d'expérience dans l'enseignement avec $30 \%$, et enfin $10 \%$ des enseignants qui ont participé à cette recherche ont une expérience comprise entre 2 et 5 ans. Ce qui signifie que nos informateurs ont l'expérience nécessaire pour pouvoir nous informer sur la didactique de la grammaire depuis plus de huit ans derniers à nos jours.

Pour le niveau d'appréciation personnelle des enseignants de français quant à la connaissance de la grammaire, le niveau le plus élevé est le niveau très bon et bon avec la représentation de $40 \%$ pour chaque niveau. Le niveau excellent et assez bon comptent respectivement $10 \%$ des participants. Ce qui signifie que la confiance en soi n'est pas très élevée puisque nous avons seulement 1 enseignant sur 10 qui croit exceller en grammaire de français et 4 qui croient que leur niveau en grammaire est très bon . Le niveau bon et assez bon qui totalise ensemble 5 répondants ne permettrait pas d'avoir confiance pendant le cours de grammaire car pour enseigner la grammaire la confiance en soi devant les élèves compte beaucoup.

Table-2: L'expérience et le degré d'appréciation chez les enseignants

\begin{tabular}{|l|l|l|l|l|l|}
\hline Expérience & Fréquence & $\mathbf{\%}$ & Degré d'appréciation & Fréquence & $\%$ \\
\hline 2-5ans & 1 & 10.0 & Excellent & 1 & 10.0 \\
\hline 6-10ans & 3 & 30.0 & Très bon & 4 & 40.0 \\
\hline 11-16ans & 6 & 60.0 & Bon & 4 & 40.0 \\
\hline Total & 10 & 100.0 & Assez bon & 1 & 10.0 \\
\hline & & Total & 10 & 100.0 \\
\hline
\end{tabular}

Source: Données collectées par questionnaire (1 pp. 42-43)

En plus de cela pour la signification de la grammaire, 83.7 des élèves interrogés définissent la grammaire comme étant un outil pour apprendre la langue, $9.0 \%$ prennent la grammaire pour une mémorisation des règles et $7.4 \%$ la considèrent comme un moyen pour la réussite à l'examen. Cela montre que la majorité des élèves veulent apprendre la grammaire ni pour mémoriser les règles ni pour réussir à l'examen de français mais pour s'en servir comme outil pour apprendre la langue en général. Quant à la place accordée à la grammaire pendant le cours de Français, pour $28.5 \%$ des élèves, la grammaire occupe une place moyenne, $27.6 \%$ des élèves affirment qu'elle a une place très importante, $23.1 \%$ disent qu'elle est importante et pour $20.8 \%$ des participants, la place de la grammaire n'est pas importante. Ceci veut dire que la majorité des élèves qui ont répondu à notre questionnaire comprennent plus ou moins l'importance de la grammaire dans le cours de français.

Table-3: La signification et la place accordées à la grammaire par les élèves

\begin{tabular}{|l|l|l|l|l|l|}
\hline $\begin{array}{l}\text { La signification } \\
\text { de la grammaire }\end{array}$ & Fréquence & $\mathbf{\%}$ & La place de la grammaire & Fréquence & \% \\
\hline Mémorisation des règles & 28 & 9.0 & Très importante & 86 & 27.6 \\
\hline Réussite aux examens & 23 & 7.3 & Importante & 72 & 23.1 \\
\hline Outil pour apprendre la langue & 261 & 83.7 & Moins importante & 89 & 28.5 \\
\hline Total & 312 & 100.0 & Pas importante & 65 & 20.8 \\
\hline & & & Total & 312 & 100.0 \\
\hline
\end{tabular}

Source: Données collectées par questionnaire (1 pp. 44-45)

De l'importance des parties qui composent le cours de grammaire $41.7 \%$ des élèves rangent en premier lieu les explications pendant le cours, en deuxième lieu vient la préparation avant le cours avec $16.3 \%$ des participants et en troisième lieu vient le contenu avec 15.1\%.Puis suivent les exercices, le travail en groupe et la compréhension par soi-même avec respectivement $12.5 \%, 8.0 \%$ et $6.4 \%$ des participants. Selon les apprenants, la partie la plus importante dans le cours de grammaire est les explications par l'enseignant. Ceci montre que le cours de grammaire est centré sur l'enseignant qui, 
fréquemment révise la démarche à suivre avant de préparer le cours avec de tels quotas: $50 \%$ des participants révisent la démarche souvent, $20 \%$ le font toujours, $20 \%$ le font quelquefois et $10 \%$ des participants révisent rarement la démarche qu'ils utiliseront pendant le cours de grammaire. Ceci signifie que la situation de la didactique de la grammaire est inquiétante car seulement 2 enseignants sur 10 se rappellent toujours de réviser la démarche qui les aidera à atteindre leurs objectifs. Cinq enseignants le font souvent et le reste ne se soucie pas beaucoup de la démarche à suivre pour la didactique de la grammaire. Cependant, il s'avère nécessaire de réviser la démarche à suivre surtout la démarche inductive et la démarche active de découverte.

Table-4: La partie du cours de grammaire la plus importante pour les élèves et la révision de la démarche par les enseignants

\begin{tabular}{|c|c|c|c|c|c|}
\hline La partie la plus importante du cours & Fréquence & $\%$ & Révision de la démarche & Fréquence & $\%$ \\
\hline Préparation avant le cours & 51 & 16.3 & Toujours & 2 & 20.0 \\
\hline Explication pendant le cours & 130 & 41.7 & Souvent & 5 & 50.0 \\
\hline Contenu & 47 & 15.1 & Quelquefois & 2 & 20.0 \\
\hline Exercices & 39 & 12.5 & Rarement & 1 & 10.0 \\
\hline compréhension par soi-même & 20 & 6.4 & Total & 10 & 100.0 \\
\hline Travail en groupe & 25 & 8.0 & & & \\
\hline Total & 312 & 100.0 & & & \\
\hline
\end{tabular}

Source: Données collectées par questionnaire (1 pp. 46-47)

En plus de l'importance qu'accordent les élèves à la grammaire et de la révision de la démarche faite par les enseignants, cette recherche nous a permis de voir pour quelle fréquence la grammaire est enseignée isolée ou intégrée dans un texte ou un autre document déclencheur. Ainsi la grammaire est enseignée isolée par $10 \%$ des participants qui le font souvent ,20\% le font quelquefois, 30\% le font rarement et $40 \%$ ne le font jamais. Ceci révère que les enseignants qui ont participé à notre recherche tendent vers l'enseignement de la grammaire contextualisé. Aussi la grammaire est-elle intégrée dans un texte ou document déclencheur pour permettre aux élèves de pouvoir contextualiser leur apprentissage. Les participants à notre recherche intègrent toujours la grammaire à $20 \%$, ils le font souvent à $50 \%$. Ceci signifie qu'au moins $70 \%$ de nos participants ont le souci d'enseigner la grammaire à partir d'un texte ou d'un document déclencheur.

Table-5: La grammaire est enseignée isolée ou intégrée dans un texte ou un autre document déclencheur

\begin{tabular}{|l|l|l|l|l|l|}
\hline Fréquence d'isolement & Fréquence & $\mathbf{\%}$ & Fréquence d'intégration & Fréquence & $\%$ \\
\hline Souvent & 1 & 10.0 & Toujours & 2 & 20.0 \\
\hline Quelquefois & 2 & 20.0 & Souvent & 5 & 50.0 \\
\hline Rarement & 3 & 30.0 & Quelquefois & 1 & 10.0 \\
\hline Jamais & 4 & 40.0 & Rarement & 1 & 10.0 \\
\hline Total & 10 & 100.0 & Jamais & 1 & 10.0 \\
\hline & & Total & 10 & 100.0 \\
\hline
\end{tabular}

Source: Données collectées par questionnaire (1 pp. 48-49)

\section{La coexistence des trois démarches}

Pour mesurer le degré de coexistence de la démarche déductive, de la démarche inductive et la démarche active de découverte, il faut questionner la connaissance que les enseignants ont de ces démarches; il sied de voir leur utilisation en classe; il convient de savoir quelle formation les enseignants ont reçue d'elles, les facteurs qui conditionnent le choix d'une telle démarche ainsi que celle qui est la plus préférée des autres démarches par les élèves.

D'abord, s'agissant de la connaissance des démarches de la didactique de la grammaire du FLE, les participants à notre recherche ont répondu qu'ils connaissent la démarche déductive avec le pourcentage le plus élevé de $50 \%$, la démarche inductive avec 30\%, la démarche active de découverte avec $10 \%$ des participants. Cela signifie que la démarche déductive est la plus connu des enseignants qui ont participé à notre recherche. Quant au degré d'utilisation de ces démarches, la recherche a montré que la démarche déductive est toujours utilisée à $30 \%$, souvent à 30\%, quelquefois à $20 \%$ et rarement à $20 \%$. La démarche inductive n'est pas toujours utilisée mais elle est souvent utilisée avec un pourcentage $70 \%$ des participants. Certains enseignants l'emploient quelquefois avec $10 \%$, rarement avec $10 \%$ et ne l'utilisent jamais avec $10 \%$ des participants. La démarche active de découverte est quelquefois utilisée avec un pourcentage culminant de $50 \%$, elle est souvent utilisée avec 10\%, rarement employée avec $20 \%$ et jamais utilisée avec $20 \%$ des participants. Cela signifie que la démarche déductive joue un grand rôle dans la pratique de tous les jours pour les enseignants du français qui ont répondu à notre questionnaire, à plus de $60 \%$ des participants. 
Table-6: Le degré d'utilisation des démarches de la didactique de la grammaire par les enseignants

\begin{tabular}{|l|l|l|l|l|l|l|l|}
\hline D. D & Fréquence & $\mathbf{\%}$ & D. I & Fréquence & $\mathbf{\%}$ & DAAD Fréquence & $\mathbf{\%}$ \\
\hline Toujours & 3 & 30.0 & Souvent & 7 & 70.0 & 1 & 10.0 \\
\hline Souvent & 3 & 30.0 & Quelque fois & 1 & 10.0 & 5 & 50.0 \\
\hline Quelquefois & 2 & 20.0 & Rarement & 1 & 10.0 & 2 & 20.0 \\
\hline Rarement & 2 & 20.0 & Jamais & 1 & 10.0 & 2 & 20.0 \\
\hline Total & 10 & 100.0 & Total & 10 & 100.0 & 10 & 100.0 \\
\hline
\end{tabular}

Source: Données collectées par questionnaire (1 pp. 51-52)

Tous les interviewés ont affirmé qu'ils connaissent la démarche déductive et la démarche inductive. Un seul participant a dit qu'il connait les trois démarches et les a apprises à l'université. Ce qui est connu de la démarche déductive. Ils caractérisent la démarche déductive de facile à appliquer pour les débutants, traditionnelle et importante pour les débutants qui doivent connaitre les règles. Toutefois, ils ajoutent que la démarche déductive limite les élèves et leur est difficile de découvrir les règles. Pour la démarche inductive, les réponses des interviewés nous révèlent que les participants n'en connaissent pas grand-chose, qu'ils ne sont pas formés dans cette démarche, que cette démarche est difficile à appliquer surtout avec les 80 minutes dont on dispose par semaine -quelque fois les 80 minutes ne sont pas successives -et qu'elle aiderait les élèves à découvrir la règle. Quant à la démarche active de découverte, les enseignants ne la connaissent pas, elle est difficile à appliquer suite à la course à la montre. Les enseignants l'utilisent rarement parce qu'il est difficile de la concilier avec le programme.

Table-7: La connaissance de la démarche à utiliser et la formation dans les nouvelles démarches

\begin{tabular}{|l|l|l|l|l|l|l|l|}
\hline Connaissance de la démarche & Fréquence & $\boldsymbol{\%}$ & Formation & $\begin{array}{l}\text { Fréquence } \\
\text { D.I }\end{array}$ & $\boldsymbol{\%}$ & $\begin{array}{l}\text { Fréquence } \\
\text { DAAD }\end{array}$ & \begin{tabular}{l} 
\% \\
\hline La démarche déductive
\end{tabular} \\
\hline La démarche inductive & 3 & 50.0 & Oui & 5 & 50.0 & 3 & 30.0 \\
\hline La démarche active de découverte & 1 & 30.0 & Non & 5 & 50.0 & 7 & 70.0 \\
\hline Total & 10 & 10.0 & Total & 10 & 100.0 & 10 & 100.0 \\
\hline
\end{tabular}

Source: Données collectées par questionnaire (1 p. 50)

Ensuite concernant la formation dans les nouvelles démarches de la didactique de la grammaire du FLE, la moitié des participants (50\%) est formée dans la démarche inductive et $50 \%$ qui reste n'en a pas reçu de formation. Quant à la démarche active de découverte, seuls $30 \%$ des participants en ont reçu une formation et $70 \%$ des participants n'en sont pas formés. Les facteurs déterminant le choix d'une démarche divergent: $60 \%$ des participants à notre recherche choisissent la démarche déductive parce qu'elle est facile à utiliser ,30\% des participants la préfèrent parce qu'ils ne sont pas formés dans d'autres démarches et $10 \%$ des participants l'utilisent parce qu'ils ne connaissent pas d'autres démarches. Aussi, cette recherche a révélé que $50 \%$ des participants choisissent la démarche inductive parce qu'elle permet d'échanger les idées, 40\% l'utilisent puis qu'elle permet de découvrir la règle facilement tandis que $10 \%$ n'en savent rien. En définitive, 30\% des enseignants qui ont répondu à notre questionnaire affirment que la démarche active de découverte (DADD) permet aux élèves de travailler en groupe, $10 \%$ ajoutent qu'elle permet aux élèves d'être actifs tandis que 30\% des participants disent qu'elle demande du temps, 20\% reviennent sur le fait qu'elle est difficile à appliquer. Cela signifie que malgré l'importance de la démarche active de découverte, les facteurs qui affectent son utilisation sont multiples. Nos interviewés ont précisé que la démarche déductive a pour avantages de permettre aux élèves de connaître l'analyse parce qu'on ne peut pas prétendre connaître la langue sans en faire l'analyse, de permettre aux enseignants de gagner du temps surtout qu'on ne dispose que de deux périodes de français par semaine et qu'elle est plus rapide.

Table-8: Les facteurs déterminant le choix d'une démarche

\begin{tabular}{|l|l|l|l|l|l|l|l|l|}
\hline Facteurs/DD & Fréq. & $\mathbf{\%}$ & Facteurs/DI & Fréq. & $\mathbf{\%}$ & $\begin{array}{l}\text { Facteurs/ } \\
\text { DAAD }\end{array}$ & Fréq. & \% \\
\hline Elle est facile & 6 & 60.0 & $\begin{array}{l}\text { Découverte } \\
\text { de la règle }\end{array}$ & 4 & 40.0 & Difficile à appliquer & 2 & 20.0 \\
\hline $\begin{array}{l}\text { Je ne connais pas d'autres } \\
\text { démarches }\end{array}$ & 1 & 10.0 & $\begin{array}{l}\text { Echanger les } \\
\text { idées }\end{array}$ & 5 & 50.0 & Elle demande du temps & 3 & 30.0 \\
\hline $\begin{array}{l}\text { Je ne suis pas formé(e) } \\
\text { dans d'autres démarches }\end{array}$ & 3 & 30.0 & Aucune idée & 1 & 10.0 & Elle rend les élèves actifs & 1 & 10.0 \\
\hline Total & 10 & 100.0 & Total & 10 & 100.0 & Suscite le travail en groupe & 3 & 30.0 \\
\hline & & & & & & On ne la connaît pas & 1 & 10.0 \\
\hline & & & & & & Total & 10 & 100.0 \\
\hline
\end{tabular}

Source: Données collectées par questionnaire (1 pp. 53-55) 
En fin, partant de la description des étapes qui composent une démarche à utiliser, nous avons constaté que $50 \%$ des participants suivent les étapes de la démarche inductive ,40\% suivent les étapes de la démarche déductive et $10 \%$ suivent les étapes de la démarche active de découverte. Ceci nous a permis de découvrir que certains enseignants suivent inconsciemment les étapes d'une démarche qu'ils ne connaissent même pas. Aussi avons-nous remarqué que parmi les trois démarches de la didactique de la grammaire française, $42.3 \%$ des élèves préfèrent la démarche déductive, $33.7 \%$ préfèrent la démarche active de découverte et $24.0 \%$ sont pour la démarche inductive. Ce qui veut dire que la démarche déductive, par la description de ses étapes, est la plus préférée par élèves.

Table-9: Les étapes suivies par les enseignants et la démarche préférée par les élèves

\begin{tabular}{|l|l|l|l|l|l|}
\hline Etapes & Fréquence & $\mathbf{\%}$ & Les démarches & Fréquence & $\%$ \\
\hline -Observation -règle -application (DD) & 4 & 40.0 & La démarche déductive & 132 & 42.3 \\
\hline -exemple-induction de la règle (DI) & 5 & 50.0 & La démarche inductive & 75 & 24.0 \\
\hline $\begin{array}{l}\text {-De l'observation au réinvestissement } \\
\text { (DAAD) }\end{array}$ & 1 & 10.0 & $\begin{array}{l}\text { La démarche active de } \\
\text { découverte }\end{array}$ & 105 & 33.7 \\
\hline Total & 10 & 100.0 & Total & 312 & 100.0 \\
\hline
\end{tabular}

Sourc: Données collectées par questionnaire (1 pp. 56-57)

\section{Attitude des enseignants de français pendant le cours de grammaire}

L'attitude des enseignants, pendant le cours de grammaire, est analysée à partir du fait que l'enseignant dicte les règles de grammaire sans considérer les réactions des élèves. Ainsi $36.5 \%$ des élèves qui ont participé à notre recherche disent que leurs enseignants ne dictent jamais les règles de grammaire sans considérer leurs réactions, $21.2 \%$ des élèves disent que leurs enseignants dictent, quelquefois, les règles de grammaire, $20.2 \%$ des élèves disent qu'ils le font toujours. Pour $12.8 \%$ des élèves, ils le font rarement et pour $9.3 \%$ de ces élèves, ils le font souvent. Ceci montre que l'attitude des enseignants de la grammaire française est bonne en ce sens qu'ils évitent à tout prix de dicter la règle de grammaire, ils tendent à leur permettre plutôt de la découvrir eux-mêmes pas à pas.
Etablissant une comparaison entre la démanche déductive, la démarche inductive et la démarche active de découverte, nos interviewés ont précisé que les démarches nouvelles sont favorables pour les élèves, que la démarche déductive permet aux enseignants d'avancer, seuls, avec la matière, que la démarche déductive est plus rapide mais n'aide pas les élèves. Ceci pousse l'enseignant à partir avec ceux qui comprennent plus rapidement et laisser les autres à côté. Les autres démarches seraient inclusives pour tous les autres élèves. Ils concluent que les nouvelles démarches sont utiles pour les élèves et permettent aux élèves de se sentir dans le cours et de s'approprier de la langue. Ils proposent - pour les démarches qui ne sont pas encore utilisées -une formation dans la pratique des nouvelles démarches pour les approfondir, une formation particulière pour la démarche active de découverte ainsi que une consultation par les paires.

Table-10: L'enseignant dicte les règles de grammaire sans considérer les réactions des élèves

\begin{tabular}{|l|l|l|}
\hline Indicateur & Fréquence & \% \\
\hline Toujours & 63 & 20.2 \\
\hline Souvent & 29 & 9.3 \\
\hline Quelquefois & 66 & 21.2 \\
\hline Rarement & 40 & 12.8 \\
\hline Jamais & 114 & 36.5 \\
\hline Total & 312 & 100.0 \\
\hline
\end{tabular}

Source: Données collectées par questionnaire (1 p. 64)

\section{CONCLUSION}

L'objectif de notre recherche était d'établir le bilan sur la pratique des démarches de la didactique de la grammaire en classe de FLE des écoles secondaires au Rwanda. Ce bilan a été établi à partir de la situation actuelle de la didactique de la grammaire en classe de FLE au Rwanda, de la coexistence des trois démarches ainsi que l'attitude des enseignants et des élèves face à cette situation de didactique de la grammaire du FLE.
Cette recherche a confirmé que la démarche déductive est la plus connue par 50\% des participants la démarche inductive suit avec $30 \%$ et vient la démarche active de découverte avec $10 \%$ des participants à notre recherche.

La démarche déductive est toujours utilisée par $30 \%$ des participants, la démarche inductive est souvent utilisée par $70 \%$ des participants tandis que la démarche active de découverte est quelquefois utilisée par $50 \%$ des participants à notre recherche. 
S'agissant de la formation dans les nouvelles démarches de la didactique de la grammaire, $50 \%$ des participants sont formés dans la démarche inductive tandis que $30 \%$ des participants sont formés dans la démarche active de découverte.

Quant aux facteurs qui influencent le choix de la démarche ,60\% des participants précisent qu'ils préfèrent la démarche déductive puisqu'elle est facile à utiliser ,50\% des participants choisissent la démarche inductive parce qu'elle permet d'échanger les idées tandis que $30 \%$ des participants expriment leur souci d'utiliser la démarche active de découverte parce qu'elle demande plus de temps. Les étapes les plus suivies par les enseignants sont pour l'induction de la règle avec $50 \%$ des participants.

Les démarches de la didactique de la grammaire les plus utilisées en classe de FLE dans les écoles secondaires du district de Kayonza, sont les démarches déductive et inductive. La démarche déductive bien qu'elle soit plus utilisée vue sa facilité et sa rapidité, elle n'est, néanmoins, favorable aux apprenants. La démarche active de découverte n'est pas connue des enseignants qui ont participé à notre recherche. Or, elle permettrait aux enseignants d'engager les élèves dans les activités de conceptualisation grammaticale. Il s'avère nécessaire de marier les trois démarches pour non seulement permettre la mémorisation, la découverte des règles grammaticales mais aussi aboutir à leur réutilisation des compétences grammaticales dans d'autres contextes.

\section{RÉFÉRENCES}

1. Rugigana, A. (2018). Les démarches de la didactique de la grammaire du FLE dans les écoles secondaires du district de Kayonza, au Rwanda(Mémoire de Master). Nairobi : Kenyatta University Library, 2018.

2. Creswell, W. J. (2008). Educational research: Planning, conducting, and evaluating quantitative and qualitative research, 3rd edition. New Jersey: Upper Saddle River.

3. Amin, M. E. (2005). Social Science Research: Conception, Methodology and Analysis. Kampala: Makerere University Printery.
4. Unil. (2010). Méthodes de recueil des données pour l'évaluation d'un cursus d'études. Lausane : Centre de Soutien à l'enseignement. 2010.

5. Lescure, R. (2010). Les approches actionnelles et par compétences en didactique du FLE : intérêts et limites; Le français de demain : enjeux éducatifs et professionnels. Colloque international. 28-30.

6. Porcher, L. (1990). L'évaluation des apprentissages en langue étrangères, Etude de linguistique appliquée. Paris: Kliencksieck, 5-39.

7. Sandra, T. (2008). L'enseignement de la grammaire dans une classe de francais au premier cycle du secondaire (Mémoire). Québec: Faculté des Sciences de l'éducation, Université Laval.

8. Nadeau, M., \& Fisher, C. (2006). La grammaire nouvelle : la comprendre et l'enseigner. Montréal, QC: Chenelière Éducation.

9. Coste, D., \& Galisson, R. (1982). Dictionnaire de didactique des langues. Paris : Hachette.

10. Perrenoud, P. (1999). L'école saisie par les compétences.. Intervention au colloque de l'Association des cadres scolaires du Québec "Former des élèves compétents : la pédagogie à la croisée des chemins", 9-11 décembre 1998. Québec : Faculté de psychologie et des sciences de l'éducation, Université de Génève.

11. Chartrand, S. G. (1996). Apprendre la grammaire par la démarche active de découverte». [auteur du livre] Chartrand, S. G. Pour un nouvel enseignement de la grammaire (2e édition). Montréal: Les Éditions Logiques, 197-225.

12. Matthews, B., \& Ross, L. (2010). Research methods: A practical guide for the social sciences. England: Harlow, Pearson.

13. Neuman, W. L. (2011). Social research methods: qualitative and quantitative approaches, 7th edition. Boston: Pearson.

14. Patton, M. Q. (2002). Qualitative research and evaluation methods. New Dheli: Sage, Publication.

15. Robert. V. K., \& Morgan, D. (1970). Determining Sample Size for Research Activities. Educational Psychological Mesearurement. 30: 607-610. 